\title{
Approximating the error probability for the independent Rayleigh fading channel
}

\author{
Jean-Claude Belfiore \\ Dept. Com, ENST \\ Paris, France. \\ E-mail: belfiore@enst.it
}

\author{
Emanuele Viterbo \\ Dipartimento di Elettronica, Politecnico di Torino, \\ C.so Duca degli Abruzzi 24, 10129, Torino, Italy. \\ E-mail: viterbo@polito.it
}

\begin{abstract}
The major contribution of this paper is the computation of an accurate approximation of the symbol error probability of multidimensional signal constellations used for transmission over independent Rayleigh fading channels. Here we attempt to compute the exact error probability and show how some apparently rather gross approximations still lead to an accurate result.
\end{abstract}

\section{INTRODUCTION}

The major contribution of this paper is the computation of an accurate approximation of the symbol error probability of multidimensional signal constellations used for transmission over independent Rayleigh fading channels. The common approach based on the union bound is known to give rather inaccurate results when dealing with large constellations. Previous work [1] showed some improvement in the union bound technique by using the exact paiwise error probability expression, but even so, the resulting upper buond is shown to be loose for large constellations. Attempts of truncating the terms in the union bound series usually lead to unpredictable results. Here we take a radically different approach: we attempt to compute the exact error probability and show how some apparently rather gross approximations still lead to an accurate result.

Let $\mathbf{x}=\left(x_{1}, \ldots, x_{n}\right)$ represent a transmitted signal vector taken from an $n$-dimensional lattice constellation $\mathcal{S}$ and consider transmision over a vector channel described by the $n \times n$ real matrix $H$. The received vector $\mathbf{y}=\left(y_{1}, \ldots, y_{n}\right)$ is given by $\mathbf{y}=\mathbf{x} H+\mathbf{z}$ where $\mathbf{z}=\left(z_{1}, \ldots, z_{n}\right)$ is a Gaussian noise vector with $z_{i}$ i.i.d., zero mean and variance $\sigma^{2}=N_{0} / 2$. The case of complex signal constellations and channels can be easily treated by separating real and imaginary parts. We consider independent Rayleigh fading channels, where $H=\operatorname{diag}\left(h_{1}, \ldots, h_{n}\right)$ and $h_{i}$ are real independent Rayleigh-distributed random variables with $E\left[h_{i}^{2}\right]=1$ and density function $f(h)=2 h e^{-h^{2}}$. The joint density function of the fading vector $\mathbf{h}=\left(h_{1}, \ldots, h_{n}\right)$ is $p(\mathbf{h})=\prod f\left(h_{i}\right)$. We assume the constellation $\mathcal{S}$ to be carved from a lattice $\Lambda=\left\{\mathbf{u} M: \mathbf{u} \in \mathbf{Z}^{n}\right\}$, defined by its generator matrix $M=\left\{v_{k j}\right\}$, so that $\mathcal{S}=\left\{\mathbf{u} M+\mathbf{x}_{0}: \mathbf{u} \in \mathcal{B} \cap \mathbf{Z}^{n}\right\}$ where $\mathcal{B}$ is usually taken to be a hypercube of edge $2^{m}-1$ to simplify the labelling operation and $\mathbf{x}_{0}$ the recentering vector which minimizes the transmited energy. Maximum likelihood decoding defines the so called Voronoi region around each lattice point $\mathbf{x}$.
Let us consider the infinite lattice case, where $\mathcal{S}=\Lambda$ and study the codeword error probability $P(e)=P(e \mid \mathbf{x}=\mathbf{0})=$ $1-P(c)$, for the simple case of independent Rayleigh fading channel. The probability of correct decision is given by

$$
P(c)=\frac{1}{(\sqrt{2 \pi} \sigma)^{n}} \int_{\mathbf{R}_{+}^{n}} \int_{\mathcal{V}(\mathbf{h})} e^{-\frac{\|\mathbf{z}\|^{2}}{2 \sigma^{2}}} p(\mathbf{h}) d \mathbf{z} d \mathbf{h}
$$

where the region $\mathcal{V}(\mathbf{h})$ is the decision region of the so called faded constellation $S_{\mathbf{h}}$ obtained by scaling the components of the code vectors by the fading coefficients. We note that $S_{\mathbf{h}}$ is a lattice costellation carved from the faded lattice having a generator matrix given by $\operatorname{diag}(\mathbf{h}) M$. Hence $\mathcal{V}(\mathbf{h})$ is always a Voronoi region of a randomly faded lattice:

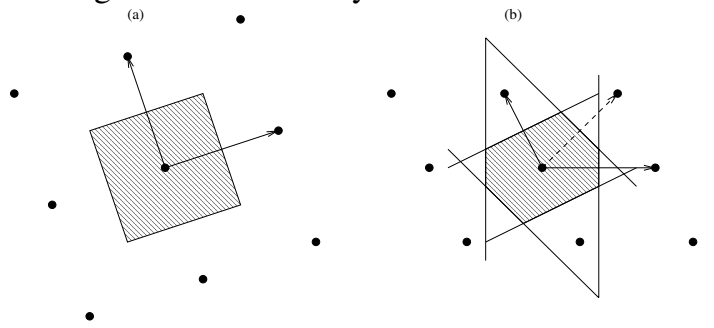

Instead of integrating the Gaussian distribution over the Voronoi cell $\mathcal{V}(\mathbf{h})$ we integrate it over a rectangular parallelotope $\mathcal{V}_{0}(\mathbf{h})$ which approximates the $\mathcal{V}(\mathbf{h})$. This yields the approximation

$$
P(e) \approx \sum_{m=1}^{2^{n}-1}(-1)^{w_{H}(m)+1} \prod_{j=1}^{n}\left(\frac{1}{1+\mu_{m j} \Gamma / 4}\right)
$$

where $\Gamma=1 / \sigma^{2}, w_{H}(m)$ is the Hamming weight of the binary representation $\left(b_{1}(m), \ldots, b_{n}(m)\right)$ of the integer $m$ and $\mu_{m, j}=\sum_{k=1}^{n} b_{k}(m) v_{k j}^{2}$. Comparisons with simulation results shows that the proposed approximation seems to be more accurate with optimally rotated cubic constellations. In this case the faded Voronoi regions appear to be better approximated with $\mathcal{V}_{0}(\mathbf{h})$ than in other cases. A precise explanation of this fenomenon is still under investigation. ${ }^{1}$

\section{REFERENCES}

[1] G. Taricco and E. Viterbo, "Performance of High-Diversity Multidimensional Costellations," IEEE Transaction on Information Theory, vol. 44, no. 4, July 1998, pp.1539-1543.

\footnotetext{
${ }^{1}$ This work was supported in part by Newcom
} 